\title{
The dairy fat paradox: Whole dairy products may be healthier than we thought
}

\author{
Sepideh Soltani ${ }^{1}$, Mohammadreza Vafa ${ }^{1 *}$ \\ Received: 17 Apr 2017 \\ Published: 18 Dec 2017
}

Copyright@ Iran University of Medical Sciences

Cite this article as: Soltani S, Vafa M. The dairy fat paradox: Whole dairy products may be healthier than we thought. Med J Islam Repub Iran. 2017 (18 Dec);31:110. https://doi.org/10.14196/mjiri.31.110

Low fat dairy products attracted attention in the 1960s because of the move against saturated fats, as it was believed that they lead to weight gain and increase LDL and cholesterol, which trigger coronary heart disease (1). In 1985, United States Department of Agriculture (USDA) recommended a switch to low-fat dairy products as an approach of controlling fat intake for everyone over the age of 2 years (1). The 2015-2020 Dietary Guidelines for Americans and the American Heart Association both suggested consuming low fat or non-fat dairy products to reduce both calorie and saturated fat intake, which could improve heart health (2). However, in recent years, scientists have called this recommendation into question $(3,4)(5)$. A large body of scientific evidence challenges those long-held beliefs that low fat dairy products are better for health. Recent studies demonstrate that high fat dairy does not cause obesity (5) or contribute to diabetes (6), or cardiovascular disease $(3,4)$; in fact, it may even help prevent them.

Consumption of dairy products as a part of current dietary guidelines is a rich source of essential micro and macronutrients, such as high-quality protein, several vitamins, and minerals (7). Dairy products have also make a large contribution to a rare source of certain fatty acids, such as short- and medium-chain saturated fatty acids and trans fatty acids (8). In the scientific community, fat consumption, especially saturated and trans fatty acids, are typically proposed as having a negative role in the development of the most common type of noncommunicable diseases, such as cardiovascular disease (CVD) (9), diabetes (10), and obesity (11).

Despite the fact that many studies have proved that dairy

Corresponding author: Dr Mohammadreza Vafa, rezavafa@yahoo.com

1. Department of Nutrition, School of Public Health, Iran University of Medical Sciences, Tehran, Iran. food reduced the risk of some types of alimentary cancers $(12,13)$, CVD (4), stroke (14), metabolic syndrome (15), and type II diabetes $(6,16)$, the findings from a series of meta-analyses remained controversial. Dairy fat is one of the most controversial components of dairy products that seems to be a major source of heterogeneity among the results from the meta-analyses that investigated the relationship between dairy products and the risk of noncommunicable diseases (5). On average, fat constitutes $3.3 \%$ to $4.4 \%$ of milk (17). A glass of whole milk contains $5 \mathrm{~g}$ saturated fatty acid, or in other words, $20 \%$ of the daily amount recommended by the 2015 Dietary Guidelines for Americans (1). Saturated fatty acid (SFA) is commonly considered to contribute to the development of CVD and stroke due to raising the ratio of LDL to HDL cholesterol (18). Therefore, dietary advisors recommend reducing the intake of full fat and high fat dairy products (2). In parallel to such recommendations, fat consumption was declined over 3 decades in the United States and many developed countries (17). Despite these attempts, reducing fat intake did not have an impact on the growing obesity, diabetes, and CVD epidemic, and thus the simplest message, which is often overlooked, is that fats do not have the same physiological effects on health (1). In fact, the current recommendations about increasing the intake of fat-free or low fat milk, and milk products in 2010 Dietary Guidelines for Americans has been based on findings from Nurses' Health Study that revealed low fat dairy intake maintained the metabolic parameters in the normal range $(19,20)$. The results of this study, which is one of the first investigations on the role of dairy fat and incidence of heart disease,

$\uparrow$ What is "already known" in this topic:

The most dietary guidelines recommending the increased consumption of low fat or fat-free dairy products.

$\rightarrow$ What this article adds:

The dairy fat intake is not typically accompanied with a higher risk of weight gain, CVD, or type II diabetes in healthy persons within the range of the recommended calorie intake. 
showed that high fat dairy consumption increased the incidence of CVD compared to the low fat dairy intake (20).

A recent meta-analysis including 22 cohort studies suggested an inverse association of whole and low fat dairy with incidence of type II diabetes, however, no significant associations were found for high fat dairy (6). In the field of heart disease and stroke, low fat dairy intake was associated with a reduced risk of total CVD and stroke $(3,4)$. In a follow-up study, despite the nonsignificant findings for consumption of full fat dairy products, the intake of low fat dairy products was found to be inversely associated with CHD risk (3). The most important findings regarding the effects of fat dairy on obesity and metabolic syndrome have been reported in a systematic review of observational studies by Kratz et al. (5). Overall, most of the included studies revealed that those participants, who consumed more dairy fat or high fat dairy foods at baseline gained less weight over time compared to participants, who consumed less (5). Furthermore, not only there was no association between consumption of dairy fat or high fat dairy foods and obesity at baseline or over time, but also low fat dairy consumption was not inversely associated with risk of obesity (5).

In most studies, FFQ was applied to the estimation of food intake, while for some foods, such as dairy fat (low fat versus high fat products), they considered less than half the inter-individual variability in consumption (21). Therefore, the objective measure of dairy fat intake includes the oddnumbered saturated fatty acids, pentadecanoic acid (C15:0), and trans palmitoleic acid, which have been validated as markers of dairy fat intake, may be considered preferable $(22,23)$. For example, pentadecanoic acid concentration has been negatively correlated with central obesity after adjustment factors, such as calcium intake and physical activity (24). In support of this finding, the results of a 5-year follow-up revealed that the pentadecanoic acid was associated with positive effects on insulin sensitivity and $\beta$ cells as well as $27 \%$ reduction in the incidence of type II diabetes (25). Moreover, the meta - analysis data from 2 large prospective cohort studies have shown that plasma pentadecanoic acid is associated with a lower incidence of diabetes by $32 \%$ and $28 \%$ in the highest plasma pentadecanoic acid versus the lowest (26). Trans palmitoleic acid is another reliable dairy fat biomarker attracting consumers' interest (22). Mozaffarian et al. found that higher trans-palmitoleate levels were associated with slightly lower adiposity, triglyceride, C-reactive protein, and insulin resistance. The finding from multiethnic US cohort indicated an inverse association for triglycerides, fasting insulin, and systolic blood pressure in individuals with Quintile 5 compared with those with Quintile 1 trans palmitoleic acid (27)

There are several points that must be considered when evaluating serum fatty acid as a biomarker of dairy fat intake. Although dairy products provide these fatty acids, they can also be derived from other forms of ruminant fat, such as beef or $\operatorname{lamb}(28,29)$. Moreover, metabolic status has also been proposed to affect certain serum fatty acids (5). Additionally, although fat constitutes a considerable proportion of dairy products, it is difficult to isolate the ef- fect of dairy fat from other dairy components that may contribute significant favorable or unfavorable effects on risk(5). It is important to note that consumption of high fat or low fat dairy products could be accompanied with other health behaviors (5). For example, high fat dairy consumption was positively associated with unhealthy lifestyle factors, such as smoking, and inversely correlated with physical activity and fiber intake (5). Additionally, different dietary sources of dairy fat may result in differential effects on health. For instance, in equal fat content, cheese showed positive effects of LDL-C compared with butter (30). The results of a recent meta-analysis of randomized clinical studies that examined the impacts of changing whole fat and low fat dairy products on cardiometabolic risk factors should also be interpreted with caution (31). As discussed by the authors, although included studies were stratified by low fat and whole fat dairy products, a direct comparison of these studies may not be considerable, as no trials have directly compared low fat dairy diet with whole fat diet (31).

In summary, the majority of evidence supports the hypothesis that dairy fat intake is not typically accompanied with an higher risk of weight gain, CVD, or type II diabetes although this is in contrast to most dietary guidelines recommending the increased consumption of low fat or fatfree dairy products. The best conclusion in dairy fat field was obtained by Dr. Mozaffarian quotation. He stated, "In the absence of any evidence for the superior effects of low fat dairy and some evidence that there may be better benefits of whole fat dairy products for diabetes, why are we recommending only low fat diary? We should be telling people to have a variety of dairy products and remove the recommendation about fat content."

\section{Conflict of Interests}

The authors declare that they have no competing interests.

\section{References}

1. Willett W. Eat, drink, and be healthy: the Harvard Medical School guide to healthy eating: Simon and Schuster; 2011.

2. United States Department of Agriculture Department of Health and Human Services. Dietary Guidelines for Americans. 2010.

3. Alexander DD, Bylsma LC, Vargas AJ, Cohen SS, Doucette A, Mohamed M, et al. Dairy consumption and CVD: a systematic review and meta-analysis. The British journal of nutrition. 2016;115(4):737-50.

4. Qin LQ, Xu JY, Han SF, Zhang ZL, Zhao YY, Szeto IM. Dairy consumption and risk of cardiovascular disease: an updated metaanalysis of prospective cohort studies. Asia Pacific journal of clinical nutrition. 2015;24(1):90-100.

5. Kratz M, Baars T, Guyenet S. The relationship between high-fat dairy consumption and obesity, cardiovascular, and metabolic disease. European journal of nutrition. 2013;52(1):1-24.

6. Gijsbers L, Ding EL, Malik VS, de Goede J, Geleijnse JM, SoedamahMuthu SS. Consumption of dairy foods and diabetes incidence: a doseresponse meta-analysis of observational studies. The American journal of clinical nutrition. 2016:ajen123216.

7. Muehlhoff E, Bennett A, McMahon D. Milk and dairy products in human nutrition: Food and Agriculture Organization of the United Nations (FAO); 2013.

8. Jensen RG. The composition of bovine milk lipids: January 1995 to December 2000. Journal of dairy science. 2002;85(2):295-350.

9. Hooper L, Summerbell CD, Higgins JPT, Thompson RL, Capps NE Smith GD, et al. Dietary fat intake and prevention of cardiovascular disease: systematic review. BMJ. 2001;322(7289):757-63. 
10. Heydemann A. An Overview of Murine High Fat Diet as a Model for Type 2 Diabetes Mellitus. Journal of Diabetes Research. 2016;2016.

11. Hill JO, Melanson EL, Wyatt HT. Dietary fat intake and regulation of energy balance: implications for obesity. The Journal of nutrition. 2000;130(2):284S-8S

12. Guo Y, Shan Z, Ren H, Chen W. Dairy consumption and gastric cancer risk: a meta-analysis of epidemiological studies. Nutrition and cancer. 2015;67(4):555-68.

13. Aune D, Lau R, Chan D, Vieira R, Greenwood D, Kampman E, et al. Dairy products and colorectal cancer risk: a systematic review and metaanalysis of cohort studies. Annals of oncology. 2012;23(1):37-45.

14. Hu D, Huang J, Wang Y, Zhang D, Qu Y. Dairy foods and risk of stroke: a meta-analysis of prospective cohort studies. Nutrition, Metabolism and Cardiovascular Diseases. 2014;24(5):460-9.

15. Chen G-C, Szeto IM, Chen L-H, Han S-F, Li Y-J, van Hekezen R, et al. Dairy products consumption and metabolic syndrome in adults: systematic review and meta-analysis of observational studies. Scientific reports. 2015;5.

16. Chen M, Sun Q, Giovannucci E, Mozaffarian D, Manson JE, Willett WC, et al. Dairy consumption and risk of type 2 diabetes: 3 cohorts of US adults and an updated meta-analysis. BMC medicine. 2014:12(1):1.

17. Jessri M, Mirmiran P, Golzarand M, Rashidkhani B, HosseiniEsfahani F, Azizi F. Comparison of trends in dietary pattern in Iran, Middle Eastern and North African countries from 1961 to 2005. Pajoohandeh Journal. 2011;16(1):1-10.

18. Hooper L, Summerbell CD, Thompson R, Sills D, Roberts FG, Moore $\mathrm{HJ}$, et al. Reduced or modified dietary fat for preventing cardiovascular disease. The Cochrane Library. 2012.

19. Sun Q, Ma J, Campos H, Hu FB. Plasma and erythrocyte biomarkers of dairy fat intake and risk of ischemic heart disease. The American Journal of Clinical Nutrition. 2007:86(4):929-37.

20. Hu FB, Stampfer MJ, Manson JE, Ascherio A, Colditz GA, Speizer FE, et al. Dietary saturated fats and their food sources in relation to the risk of coronary heart disease in women. The American journal of clinical nutrition. 1999;70(6):1001-8.

21. Salvini S, Hunter DJ, Sampson L, Stampfer MJ, Colditz GA, Rosner $\mathrm{B}$, et al. Food-based validation of a dietary questionnaire: the effects of week-to-week variation in food consumption. International journal of epidemiology. 1989;18(4):858-67.

22. Wolk A, Vessby B, Ljung H, Barrefors P. Evaluation of a biological marker of dairy fat intake. The American journal of clinical nutrition. 1998;68(2):291-5

23. Micha R, King IB, Lemaitre RN, Rimm EB, Sacks F, Song X, et al Food sources of individual plasma phospholipid trans fatty acid isomers: the Cardiovascular Health Study. The American journal of clinical nutrition. 2010;91(4):883-93.

24. Smedman AE, Gustafsson I-B, Berglund LG, Vessby BO. Pentadecanoic acid in serum as a marker for intake of milk fat: relations between intake of milk fat and metabolic risk factors. The American journal of clinical nutrition. 1999;69(1):22-9.

25. Santaren ID, Watkins SM, Liese AD, Wagenknecht LE, Rewers MJ, Haffner SM, et al. Serum pentadecanoic acid (15: 0), a short-term marker of dairy food intake, is inversely associated with incident type 2 diabetes and its underlying disorders. The American journal of clinical nutrition. 2014;100(6):1532-40.

26. Mozaffarian D, de Oliveira Otto MC, Lemaitre RN, Fretts AM, Hotamisligil G, Tsai MY, et al. trans-Palmitoleic acid, other dairy fat biomarkers, and incident diabetes: the Multi-Ethnic Study of Atherosclerosis (MESA). The American journal of clinical nutrition. 2013;97(4):854-61.

27. Mozaffarian D, Cao H, King IB, Lemaitre RN, Song X, Siscovick DS, et al. Trans-palmitoleic acid, metabolic risk factors, and new-onset diabetes in US adults: a cohort study. Annals of internal medicine. 2010;153(12):790-9.

28. Koger T, Wulf D, Weaver A, Wright C, Tjardes K, Mateo K, et al. Influence of feeding various quantities of wet and dry distillers grains to finishing steers on carcass characteristics, meat quality, retail-case life of ground beef, and fatty acid profile of longissimus muscle. Journal of animal science. 2010;88(10):3399-408

29. Özogul Y, Özogul Fh, Çi çek E, Polat A, Kuley E. Fat content and fatty acid compositions of 34 marine water fish species from the Mediterranean Sea. International journal of food sciences and nutrition. 2009;60(6):464-75.

30. Huth PJ, Park KM. Influence of dairy product and milk fat consumption on cardiovascular disease risk: a review of the evidence.
Advances in Nutrition: An International Review Journal. 2012;3(3):266-85.

31. Benatar JR, Sidhu K, Stewart RA. Effects of high and low fat dairy food on cardio-metabolic risk factors: a meta-analysis of randomized studies. PLoS One. 2013;8(10):e76480. 